PROCEEDINGS OF THE

AMERICAN MATHEMATICAL SOCIETY

Volume 137, Number 7, July 2009, Pages 2251-2264

S 0002-9939(08)09798-0

Article electronically published on December 23, 2008

\title{
INFINITELY GENERATED LAWSON HOMOLOGY GROUPS ON SOME RATIONAL PROJECTIVE VARIETIES
}

\author{
WENCHUAN HU \\ (Communicated by Daniel Ruberman)
}

\begin{abstract}
We construct rational projective 4-dimensional varieties with the property that certain Lawson homology groups tensored with $\mathbb{Q}$ are infinite dimensional $\mathbb{Q}$-vector spaces. More generally, for each pair of integers $p$ and $k$, with $k \geq 0, p>0$, we find a projective variety $Y$ such that $L_{p} H_{2 p+k}(Y)$ is infinitely generated.

We also construct two singular rational projective 3-dimensional varieties $Y$ and $Y^{\prime}$ with the same homeomorphism type but different Lawson homology groups; specifically, $L_{1} H_{3}(Y)$ is not isomorphic to $L_{1} H_{3}\left(Y^{\prime}\right)$ even up to torsion.
\end{abstract}

\section{INTRODUCTION}

This paper gives examples of singular rational projective 4-dimensional varieties with infinitely generated Lawson homology groups even modulo torsion. This is totally different from the smooth case ([14], also [8]), where it is known that all Lawson homology groups of rational fourfolds are finitely generated.

This paper also gives examples of singular rational projective 3-dimensional varieties with the same homeomorphism type but different Lawson homology groups.

For an algebraic variety $X$ over $\mathbb{C}$, the Lawson homology $L_{p} H_{k}(X)$ of $p$-cycles is defined by

$$
L_{p} H_{k}(X):=\pi_{k-2 p}\left(\mathcal{Z}_{p}(X)\right), \quad k \geq 2 p \geq 0,
$$

where $\mathcal{Z}_{p}(X)$ is provided with a natural topology. For general background, the reader is referred to the survey paper [12].

Clemens showed that the Griffiths group of 1-cycles (which is defined to be the group of algebraic 1-cycles homologically equivalent to zero modulo l-cycles algebraically equivalent to zero) may be infinitely generated even modulo the torsion elements for general quintic hypersurfaces in $\mathbb{P}^{4}$ (cf. [2]). E. Friedlander showed that $L_{1} H_{2}(X)$ is exactly the algebraic 1-cycles modulo algebraic equivalence (cf. [5]). Hence the Griffiths group of 1-cycles for $X$ is a subgroup of $L_{1} H_{2}(X)$.

This leads to the following question:

(Q): Can one show that $L_{p} H_{2 p+j}(X)$ is not finitely generated for some projective variety $X$ where $j>0$ ?

Received by the editors April 2, 2007, and, in revised form, October 9, 2008.

2000 Mathematics Subject Classification. Primary 14F43; Secondary 55Pxx.

Key words and phrases. Lawson homology, infinitely generated, non-homeomorphic invariants.

(C) 2008 by the author 
In this paper we shall construct, for any given integers $p$ and $j>0$, examples of rational varieties $X$ for which $L_{p} H_{2 p+j}(X)$, as an abelian group, is infinitely generated. Thus, we answer affirmatively the above question:

Theorem 1.1. There exists a rational projective variety $X$ with $\operatorname{dim}(X)=4$ such that $L_{1} H_{3}(X) \otimes \mathbb{Q}$ is not a finite dimensional $\mathbb{Q}$-vector space.

By using the projective bundle theorem given by Friedlander and Gabber ([6]), we have the following corollary:

Corollary 1.2. For any $p \geq 1$, there exists a projective algebraic variety $X$ such that $L_{p} H_{2 p+1}(X)$ is not a finitely generated abelian group.

More generally, we have

Theorem 1.3. For integers $p$ and $k$, with $k \geq 0, p>0$, we can find a projective variety $Y$ such that $L_{p} H_{2 p+k}(Y)$ is infinitely generated.

Remark 1.4. The lack of smoothness is essential here. Compare Theorem 1.1 with the following result proved by C. Peters.

Theorem 1.5 (Peters [14). For any smooth projective variety $X$ over $\mathbb{C}$ with $\mathrm{Ch}_{0}(X) \otimes \mathbb{Q} \cong \mathbb{Q}$, the natural map $\Phi: L_{1} H_{*}(X) \otimes \mathbb{Q} \rightarrow H_{*}(X, \mathbb{Q})$ is injective. In particular, $L_{1} H_{*}(X) \otimes \mathbb{Q}$ is a finite dimensional $\mathbb{Q}$-vector space.

Note that a rational variety $X$ (smooth or not) has the property that $\mathrm{Ch}_{0}(X) \otimes$ $\mathbb{Q} \cong \mathbb{Q}$. This property holds on a more general class of varieties called rationally connected varieties (cf. [10]), although it was known much earlier on rational varieties.

Applying the same construction to hypersurfaces in $\mathbb{P}^{3}$, we obtain the following:

Theorem 1.6. There exist two rational 3-dimensional projective varieties $Y$ and $Y^{\prime}$ which are homeomorphic but for which the Lawson homology groups $L_{1} H_{3}(Y)$ and $L_{1} H_{3}\left(Y^{\prime}\right)$ are not isomorphic even up to torsion.

Remark 1.7. In fact, the varieties in Theorem 1.6 have exactly one isolated singular point.

Remark 1.8. As in Theorem 1.3, the existence of a singular point of $Y$ and $Y^{\prime}$ in Theorem 1.6 is essential. If $Y, Y^{\prime}$ are two smooth rational projective varieties, then $L_{1} H_{3}(Y) \cong L_{1} H_{3}\left(Y^{\prime}\right)$ and hence $L_{1} H_{3}(Y, \mathbb{Q}) \cong L_{1} H_{3}\left(Y^{\prime}, \mathbb{Q}\right)$.

\section{LAWSON HOMOLOGY}

In this section we briefly review the definitions and results used in the next section. Let $X$ be a projective variety of dimension $m$ over $\mathbb{C}$. The group of $p$ cycles on $X$ is the free abelian group $\mathcal{Z}_{p}(X)$ generated by irreducible $p$-dimensional subvarieties.

Definition 2.1. The Lawson homology $L_{p} H_{k}(X)$ of $p$-cycles on $X$ is defined by

$$
L_{p} H_{k}(X):=\pi_{k-2 p}\left(\mathcal{Z}_{p}(X)\right), \quad k \geq 2 p \geq 0,
$$

where $\mathcal{Z}_{p}(X)$ is provided with a natural, compactly generated topology (cf. [5], [1] and [12]). 
Definition 2.2. The Griffiths group $\operatorname{Griff}_{p}(X)$ of $p$-cycles on $X$ is defined by

$$
\operatorname{Griff}_{p}(X):=\mathcal{Z}_{p}(X)_{h o m} / \mathcal{Z}_{p}(X)_{a l g},
$$

where $\mathcal{Z}_{p}(X)_{h o m}$ denotes algebraic $p$-cycles homologous to zero and $\mathcal{Z}_{p}(X)_{\text {alg }}$ denotes algebraic $p$-cycles which are algebraically equivalent to zero.

Remark 2.3. It was shown by Friedlander that $L_{p} H_{2 p}(X) \cong \mathcal{Z}_{p}(X) / \mathcal{Z}_{p}(X)_{\text {alg }}$ (cf. [5]). Hence the Griffiths group $\operatorname{Griff}_{p}(X)$ is a subgroup of the Lawson homology $L_{p} H_{2 p}(X)$. Therefore, for any projective variety $X$ (note that its homology groups are finitely generated), $\operatorname{Griff}_{p}(X)$ is infinitely generated if and only if $L_{p} H_{2 p}(X)$ is.

Remark 2.4. For a quasi-projective variety $U, L_{p} H_{k}(U)$ is also well-defined and independent of the projective embedding (cf. [13], [12]).

Let $V \subset U$ be a Zariski open subset of a quasi-projective variety $U$. Set $Z=$ $U-V$. Then we have

Theorem 2.5 (13]). There is a long exact sequence for the pair $(U, Z)$, i.e.,

$$
\cdots \rightarrow L_{p} H_{k}(Z) \rightarrow L_{p} H_{k}(U) \rightarrow L_{p} H_{k}(V) \rightarrow L_{p} H_{k-1}(Z) \rightarrow \cdots .
$$

Remark 2.6. For any quasi-projective variety $U, L_{0} H_{k}(U) \cong H_{k}^{B M}(U)$, where $H_{k}^{B M}(U)$ is the Borel-Moore homology. This follows from the Dold-Thom Theorem [3].

As a direct application of this long exact sequence, one has the following results 13]:

Proposition 2.7. $\quad$ (a) By the Complex Suspension Theorem [11, we have

$$
\begin{cases}L_{p} H_{2 n}\left(\mathbb{C}^{n}\right)=\mathbb{Z}, & \text { if } 0 \leq p \leq n \\ L_{p} H_{k}\left(\mathbb{C}^{n}\right)=0, & \text { if } 0 \leq 2 p \leq k \neq 2 n .\end{cases}
$$

(b) Let $U=\mathbb{C}^{n}$ and $V_{n-1} \subset \mathbb{C}^{n}$ be a closed algebraic set. Set $V_{n}=\mathbb{C}^{n}-V_{n-1}$. Then we have

$0 \rightarrow L_{p} H_{2 n+1}\left(V_{n}\right) \rightarrow L_{p} H_{2 n}\left(V_{n-1}\right) \rightarrow L_{p} H_{2 n}\left(\mathbb{C}^{n}\right) \rightarrow L_{p} H_{2 n}\left(V_{n}\right) \rightarrow L_{p} H_{2 n-1}\left(V_{n-1}\right) \rightarrow 0$

and

$$
L_{p} H_{k+1}\left(V_{n}\right) \cong L_{p} H_{k}\left(V_{n-1}\right), \quad k \neq 2 n, 2 n+1
$$

\section{An ELEMENTARY CONSTRUCtion}

Construction: Let $X=\left(f\left(x_{0}, \cdots, x_{n+1}\right)=0\right)$ be a smooth hypersurface in $\mathbb{P}^{n+1}$ with degree $d$ such that $X \cap\left(x_{0}=0\right)$ is smooth, and let $V_{n}:=X-X \cap\left\{\mathbb{P}^{n}=\right.$ $\left.\left(x_{0}=0\right)\right\}$ be the affine part, i.e. $V_{n} \subset \mathbb{C}^{n+1}$. Define $V_{n+1}:=\mathbb{C}^{n+1}-V_{n}$. Then $V_{n+1}$ can be viewed as an affine variety in $\mathbb{C}^{n+2}$ defined by $x_{n+2} \cdot f\left(1, x_{1}, \cdots, x_{n+1}\right)-1=$ 0 , where $V_{n}=\left(f\left(1, x_{1}, \cdots, x_{n+1}\right)=0\right)$. Denote by $\overline{V_{n+1}}$ the projective closure of $V_{n+1}$ in $\mathbb{P}^{n+2}$ and set $Z_{n}=\overline{V_{n+1}}-V_{n+1}$. Note that $\overline{V_{n+1}} \subset \mathbb{P}^{n+2}$ is given by $x_{n+2} \cdot f\left(x_{0}, x_{1}, \cdots, x_{n+1}\right)-x_{0}^{d+1}=0$. The construction here makes it clear how to use the localization exact sequence (cf. Theorem 2.5).

We leave the study of this case, where $n=1$ and $X$ is a smooth plane curve, as an exercise. 
3.1. Application to the case $n=2$. In this subsection, we will show that there exist two rational projective 3-dimensional varieties with the same singular homology groups but different Lawson homology.

The following result proved by Friedlander will be used several times:

Theorem 3.1 (Friedlander [5]). Let $X$ be any smooth projective variety of dimension $n$. Then we have the following isomorphisms:

$$
\left\{\begin{array}{l}
L_{n-1} H_{2 n}(X) \cong \mathbb{Z} \\
L_{n-1} H_{2 n-1}(X) \cong H_{2 n-1}(X, \mathbb{Z}) \\
L_{n-1} H_{2 n-2}(X) \cong H_{n-1, n-1}(X, \mathbb{Z})=N S(X), \\
L_{n-1} H_{k}(X)=0 \text { for } k>2 n .
\end{array}\right.
$$

For a finitely generated abelian group $G$, we denote by $\operatorname{rk}(G)$ the $\operatorname{rank}$ of $G$.

Let $X \subset \mathbb{P}^{3}$ be a smooth surface with degree $d=4$ such that $C:=X \cap \mathbb{P}^{2}$ is a smooth curve in $\mathbb{P}^{2}$. Then $V_{2}=X-X \cap \mathbb{P}^{2}$.

Lemma 3.2. $\operatorname{rk}\left(L_{1} H_{2}(X)\right)=\operatorname{rk}\left(L_{1} H_{2}\left(V_{2}\right)\right)+1 ; \operatorname{rk}\left(L_{1} H_{3}\left(V_{2}\right)\right)=0$.

Proof. Note that $X$ is a smooth hypersurface in $\mathbb{P}^{3}$ and so $H_{3}(X)=0$ by the Weak Lefschetz Theorem. By Theorem [3.1, we get $L_{1} H_{3}(X) \cong H_{3}(X)=0$. Now by applying Theorem 2.5 to the pair $(X, C)$, we get

$$
0 \rightarrow L_{1} H_{3}\left(V_{2}\right) \rightarrow L_{1} H_{2}(C) \rightarrow L_{1} H_{2}(X) \rightarrow L_{1} H_{2}\left(V_{2}\right) \rightarrow 0 .
$$

Note that $L_{1} H_{2}(C) \cong \mathbb{Z}$ and the map $L_{1} H_{2}(C) \rightarrow L_{1} H_{2}(X)$ is injective because the composition of it with $L_{1} H_{2}(X) \rightarrow L_{1} H_{2}\left(\mathbb{P}^{3}\right)=\mathbb{Z}$ is the degree map. So we get $L_{1} H_{3}\left(V_{2}\right)=0$. Therefore, by the above long exact sequence, we have $\operatorname{rk}\left(L_{1} H_{2}(X)\right)=\operatorname{rk}\left(L_{1} H_{2}\left(V_{2}\right)\right)+1$.

Lemma 3.3. $\operatorname{rk}\left(L_{1} H_{2}\left(Z_{2}\right)\right)=1 ; \operatorname{rk}\left(L_{1} H_{3}\left(Z_{2}\right)\right)=6 ; \operatorname{rk}\left(L_{1} H_{4}\left(Z_{2}\right)\right)=2$.

Proof. Note that $Z_{2}=\overline{V_{3}}-V_{3}$ is defined by $\left(x_{4} \cdot f\left(0, x_{1}, \ldots, x_{3}\right)=0, x_{0}=0\right)$ in $\mathbb{P}^{4}$. Let $C^{\prime}=\left(x_{4}=0\right) \cap\left(f\left(0, x_{1}, \cdots, x_{3}\right)=0\right)$ in the hyperplane $\left(x_{0}=0\right) \subset \mathbb{P}^{3}$. It is easy to see that $C^{\prime} \cong C$. Then $Z_{2}=\mathbb{P}^{2} \cup \Sigma C$, where $\Sigma C$ means the join of $C$ and the point $p=[0: \cdots: 0: 1]$. By applying Theorem 2.5 to the pair $\left(Z_{2}, \Sigma C\right)$, we get $(3.1) \cdots \rightarrow L_{1} H_{3}\left(Z_{2}-\Sigma C\right) \rightarrow L_{1} H_{2}(\Sigma C) \rightarrow L_{1} H_{2}\left(Z_{2}\right) \rightarrow L_{1} H_{2}\left(Z_{2}-\Sigma C\right) \rightarrow 0$.

Note that $Z_{2}-\Sigma C \cong \mathbb{P}^{2}-C, L_{1} H_{3}\left(\mathbb{P}^{2}-C\right)=0$ and $L_{1} H_{2}\left(\mathbb{P}^{2}-C\right)=0$. So $L_{1} H_{3}\left(Z_{2}-\Sigma C\right)=0$ and $L_{1} H_{2}\left(Z_{2}-\Sigma C\right)=0$. Therefore $\operatorname{rk}\left(L_{1} H_{2}\left(Z_{2}\right)\right)=$ $\operatorname{rk} L_{1} H_{2}(\Sigma C)=1$ by Equation (3.1).

By applying Theorem 2.5 to the pair $\left(Z_{2}, \mathbb{P}^{2}\right)$, we get

$$
\cdots \rightarrow L_{1} H_{4}\left(\mathbb{P}^{2}\right) \rightarrow L_{1} H_{4}\left(Z_{2}\right) \rightarrow L_{1} H_{4}\left(Z_{2}-\mathbb{P}^{2}\right) \rightarrow L_{1} H_{3}\left(\mathbb{P}^{2}\right) \rightarrow \cdots .
$$

Since $Z_{2}-\mathbb{P}^{2} \cong \Sigma C-C$, we have $L_{1} H_{5}\left(Z_{2}-\mathbb{P}^{2}\right) \cong L_{1} H_{5}(\Sigma C-C)=0$ and $L_{1} H_{4}\left(Z_{2}-\mathbb{P}^{2}\right) \cong L_{1} H_{4}(\Sigma C-C) \cong \mathbb{Z}$. Note that $L_{1} H_{4}\left(\mathbb{P}^{2}\right) \cong H_{4}\left(\mathbb{P}^{2}\right) \cong \mathbb{Z}$ and $L_{1} H_{3}\left(\mathbb{P}^{2}\right) \cong H_{3}\left(\mathbb{P}^{2}\right)=0$. Hence the last statement follows from Equation (3.2).

Recall that the Complex Suspension Theorem says that the suspension map $\Sigma: Y \rightarrow \Sigma Y$ induces an isomorphism $L_{m} H_{k}(Y) \cong L_{m+1} H_{k+2}(\Sigma Y)$ for $k \geq 2 m \geq 0$ and any complex projective variety (not necessarily smooth or irreducible). In particular, we have $L_{1} H_{3}(\Sigma C) \cong L_{0} H_{1}(C)$. By the Dold-Thom Theorem, we have $L_{0} H_{1}(C) \cong H_{1}(C)$. By assumption, $C$ is a smooth plane curve of degree 4 . The adjunction formula gives $\operatorname{rk}\left(H_{1}(C)\right)=6$. The second statement follows from Equation (3.1). 
Lemma 3.4. $\operatorname{rk}\left(L_{1} H_{2}\left(\overline{V_{3}}\right)\right) \leq 1 ; \operatorname{rk}\left(L_{1} H_{3}\left(\overline{V_{3}}\right)\right)=\operatorname{rk}\left(L_{1} H_{2}(X)\right)+\operatorname{rk}\left(L_{1} H_{2}\left(\overline{V_{3}}\right)\right)+4$.

Proof. Applying Theorem 2.5 to the pair $\left(\overline{V_{3}}, Z_{2}\right)$ with $p=1$, we have

$$
0 \rightarrow L_{1} H_{3}\left(Z_{2}\right) \rightarrow L_{1} H_{3}\left(\overline{V_{3}}\right) \rightarrow L_{1} H_{3}\left(V_{3}\right) \rightarrow L_{1} H_{2}\left(Z_{2}\right) \rightarrow L_{1} H_{2}\left(\overline{V_{3}}\right) \rightarrow 0
$$

since Lemma 3.2 gives $L_{1} H_{2}\left(V_{3}\right)=0$ and Proposition 2.7 gives $L_{1} H_{4}\left(V_{3}\right) \cong$ $L_{1} H_{3}\left(V_{2}\right)=0$. Hence $\operatorname{rk}\left(L_{1} H_{2}\left(\overline{V_{3}}\right)\right) \leq 1$ by Lemma 3.3. Moreover, we have $\operatorname{rk}\left(L_{1} H_{3}\left(Z_{2}\right)\right)-\operatorname{rk}\left(L_{1} H_{3}\left(\overline{V_{3}}\right)\right)+\operatorname{rk}\left(L_{1} H_{3}\left(V_{3}\right)\right)-\operatorname{rk}\left(L_{1} H_{2}\left(Z_{2}\right)\right)+\operatorname{rk}\left(L_{1} H_{2}\left(\overline{V_{3}}\right)\right)=0$. By Lemmas 3.2 and 3.3 and part (b) in Proposition 2.7, we get

$$
6-\operatorname{rk}\left(L_{1} H_{3}\left(\overline{V_{3}}\right)\right)+\left(\operatorname{rk}\left(L_{1} H_{2}(X)\right)-1\right)-1+\operatorname{rk}\left(L_{1} H_{2}\left(\overline{V_{3}}\right)\right)=0
$$

and the second formula follows.

Lemma 3.5. $\operatorname{Sing}\left(\overline{V_{3}}\right) \cong\left\{X \cap\left(x_{0}=0\right)\right\} \cup\{p\} \cong C \cup\{p\}$, where $p=[0: \cdots 0: 1]$.

Proof. The proof follows from a direct computation. By definition,

$$
\begin{gathered}
\operatorname{Sing}\left(\overline{V_{3}}\right)=\left\{\begin{array}{l}
F\left(x_{0}, x_{1}, x_{2}, x_{3}, x_{4}\right)=0, \\
d F\left(x_{0}, x_{1}, x_{2}, x_{3}, x_{4}\right)=0
\end{array}\right\} \\
=\left\{\begin{array}{l}
x_{0}^{d+1}-x_{4} \cdot f\left(x_{0}, x_{1}, x_{2}, x_{3}\right)=0, \\
(d+1) x_{0}-x_{4} \cdot \frac{\partial f}{\partial x_{0}}=0, \\
-x_{4} \cdot \frac{\partial f}{\partial x_{1}}=0, \\
-x_{4} \cdot \frac{\partial f}{\partial x_{2}}=0, \\
-x_{4} \cdot \frac{\partial f}{\partial x_{3}}=0, \\
f\left(x_{0}, x_{1}, x_{2}, x_{3}\right)=0
\end{array}\right\} \\
=\left\{\begin{array}{l}
x_{0}=0, \\
x_{4} \cdot \frac{\partial f}{\partial x_{0}}=0, \\
x_{4} \cdot \frac{\partial f}{\partial x_{1}}=0, \\
x_{4} \cdot \frac{\partial f}{\partial x_{2}}=0, \\
x_{4} \cdot \frac{\partial f}{\partial x_{3}}=0, \\
f\left(x_{0}, x_{1}, x_{2}, x_{3}\right)=0
\end{array}\right\} \\
=\left\{x_{0}=x_{4}=f\left(x_{0}, x_{1}, x_{2}, x_{3}\right)=0\right\} \cup\left\{x_{0}=f=\frac{\partial f}{\partial x_{0}}=\frac{\partial f}{\partial x_{1}}=\frac{\partial f}{\partial x_{2}}=\frac{\partial f}{\partial x_{3}}=0\right\} \\
\cong\left\{x_{0}=x_{4}=f\left(x_{0}, x_{1}, x_{2}, x_{3}\right)=0\right\} \cup\{p\} \cong C \cup\{p\} \\
0.00
\end{gathered}
$$

since $C=(f=0)$ is smooth by our assumption.

Remark 3.6. Note that $p$ is an isolated singular point and the singularity $C=$ $X \cap\left(x_{0}=0\right)$ is of $A_{4}$-type since $\operatorname{deg} X=4$. From the computation in the proof of Lemma 3.5, we know $p$ is not on $C$. We can resolve the singularity of the $A_{4^{-}}$ type part by blowing up twice over the singularity, i.e., by blowing up over the singularity for the first time and then blowing up the singularity of the proper transform of the first blowup (cf. [4]). We denote by $\widetilde{V_{3}}$ the proper transform of $\overline{V_{3}}$ with the exceptional divisor $D_{1}$ for the first blowup and by $\underset{\widetilde{V_{3}}}{\stackrel{2}{\sigma}}$ the proper transform of $\widetilde{\bar{V}_{3}}$ with the exceptional divisor $D_{2}$ for the second blowup. Both $D_{1}$ and $D_{2}$ are isomorphic to a fiber bundle over $C$ with fibre the union of two $\mathbb{P}^{1}$ intersecting at exactly one point. See the appendix for the computation of a concrete example.

Now $\underset{\overline{V_{3}}}{\approx}$ has only one singular point, denoted by $q$. 
Lemma 3.7. The singular point $q$ in $\widetilde{\widetilde{V_{3}}}$ can be resolved by one blowup whose exceptional divisor is isomorphic to $X$.

Proof. Note that $\underset{\widetilde{V_{3}}}{\widetilde{a}}$ is obtained by taking the proper transform of blowups away from $q$ and so the singularity type of $q$ is exactly the same as that of $p$ in $\overline{V_{3}}$. In the following we focus on the resolution of the singularity for $\overline{V_{3}}$ at $p$. We need to show that the proper transform of $\overline{V_{3}}$ under the blowup of $\mathbb{P}^{4}$ at $p$ is smooth away from the 1-dimensional singularity and the exceptional divisor is isomorphic to $X$.

Recall that $\overline{V_{3}} \subset \mathbb{P}^{4}$ is defined by a single equation $x_{4} \cdot f\left(x_{0}, x_{1}, x_{2}, x_{3}\right)-x_{0}^{5}=0$ and $p=[0: 0: 0: 0: 1]$. Hence locally, $p$ is in the open affine subset $\left(x_{4} \neq 0\right) \subset \mathbb{P}^{4}$. Note that $\left(x_{4} \neq 0\right) \cong \mathbb{C}^{4}$ with affine coordinates $\left(x_{0}, x_{1}, x_{2}, x_{3}\right)$. Then $p$ is the origin of $\mathbb{C}^{4}$ and the defining equation of $\overline{V_{3}}$ in $\left(x_{4} \neq 0\right)=\mathbb{C}^{4}$ is $f\left(x_{0}, x_{1}, x_{2}, x_{3}\right)-x_{0}^{5}=0$. Set $U_{3}:=\overline{V_{3}} \cap\left(x_{4} \neq 0\right)$.

Let $\widetilde{\mathbb{C}}^{4}$ be the blowup of $\mathbb{C}^{4}$ at the origin. Hence $\widetilde{\mathbb{C}}^{4}$ is given by the system of equations $\left(x_{i} y_{j}=x_{j} y_{i}, 0 \leq i \neq j \leq 3\right)$ in $\mathbb{C}^{4} \times \mathbb{P}^{3}$, where $\left[y_{0}, y_{1}, y_{2}, y_{3}\right]$ are the homogeneous coordinates of $\mathbb{P}^{3}$. Hence the inverse image $\rho^{-1}\left(U_{3}\right)$ of $U_{3}$ under the blowup $\rho: \widetilde{\mathbb{C}}^{4} \rightarrow \mathbb{C}^{4}$ is given by the system of equations

$$
\left\{\begin{array}{l}
x_{i} y_{j}=x_{j} y_{i}, 0 \leq i \neq j \leq 3 \\
f\left(x_{0}, x_{1}, x_{2}, x_{3}\right)-x_{0}^{5}=0 .
\end{array}\right.
$$

Note that $\widetilde{\mathbb{C}}^{4}$ is covered by 4 affine parts: $\left(y_{i} \neq 0\right), i=0,1,2,3$. On $\left(y_{0} \neq 0\right)$, the defining equations for $\widetilde{\mathbb{C}}^{4}$ are simplified to be the system of equations

$$
x_{i}=x_{0} y_{i}, \quad i=1,2,3,
$$

and $\rho^{-1}\left(U_{3}\right)$ in $\left(y_{0} \neq 0\right)$ is given by

$$
\left\{\begin{array}{l}
x_{i}=x_{0} y_{i}, i=1,2,3 \\
f\left(x_{0}, x_{1}, x_{2}, x_{3}\right)-x_{0}^{5}=0 .
\end{array}\right.
$$

Plug in $x_{i}=x_{0} y_{i}, i=1,2,3$, to the equation $f\left(x_{0}, x_{1}, x_{2}, x_{3}\right)-x_{0}^{5}=0$. Then we get $f\left(x_{0}, x_{0} y_{1}, x_{0} y_{2}, x_{0} y_{3}\right)-x_{0}^{5}=0$; i.e., $x_{0}^{4} \cdot f\left(1, y_{1}, y_{2}, y_{3}\right)-x_{0}^{5}=0$ since $f$ is homogeneous of degree 4 . So either $x_{0}^{4}=0$ or $f\left(1, y_{1}, y_{2}, y_{3}\right)-x_{0}=0$. Therefore the proper transform of $U_{3}$ on the affine part $\left(y_{0} \neq 0\right)$ is defined by

$$
\left\{\begin{array}{l}
x_{i}=x_{0} y_{i}, i=1,2,3 \\
f\left(1, y_{1}, y_{2}, y_{3}\right)-x_{0}=0,
\end{array}\right.
$$

for which the smoothness is easy to see from the definition and the choice of $f$.

The exceptional divisor is defined by

$$
\left\{\begin{array}{l}
x_{i}=x_{0} y_{i}, i=1,2,3, \\
f\left(1, y_{1}, y_{2}, y_{3}\right)-x_{0}=0, \\
x_{0}^{4}=0,
\end{array}\right.
$$

which is equivalent to $f\left(1, y_{1}, y_{2}, y_{3}\right)=0$ in $0 \times\left(y_{0} \neq 0\right) \subset \mathbb{C}^{4} \times \mathbb{P}^{3}$. This is the affine part of $X$ in $\mathbb{P}^{3}$. The computation on other open affine parts is the same as above. This completes the proof of the lemma.

We denote by $W_{3}$ the proper transform of the blowup in the above lemma. Note that $W_{3}$ is a smooth rational threefold. We have the following property on $W_{3}$ : 
Proposition 3.8. For a smooth surface $X \subset \mathbb{P}^{3}$ of fixed degree $d=4$ such that $X \cap\left(x_{0}=0\right)$ is smooth, the $W_{3}$ thus constructed is a smooth rational threefold with a fixed homeomorphic type; i.e., for two smooth surfaces $X$ and $X^{\prime}$ of the same degree $d$ in $\mathbb{P}^{3}$, the corresponding smooth rational threefolds $W_{3}$ and $W_{3}^{\prime}$ are homeomorphic.

Proof. Note that $\bar{V}_{3}$ is a hypersurface in $\mathbb{P}^{4}$. Let $\left(F_{t}\left(x_{0}, \cdots, x_{4}\right)=0\right) \subset \mathbb{P}^{4}$ be a family of hypersurfaces such that $\bar{V}_{3}=\left(F_{0}=0\right)$ is transversal to the hypersurface $H=\left(x_{0}=0\right)$. Let $\Delta$ be a neighborhood of $t=0$ such that $\left(F_{t}=0\right)$ is transversal to $H$ for all $t \in \Delta$. Let $\Gamma \subset \mathbb{P}^{4} \times \Delta$ be the (analytic) variety defined by $F(x, t):=$ $F_{t}(x)=0$. Then we have the following incidence correspondence:



with $\pi^{-1}(t) \cap \Gamma=\Gamma^{t}$.

The same type of computation in Remark 3.6 and Lemma 3.7 works for a family of singularities. The reader is referred to a more general discussion for the simultaneous resolution of rational singularities to [1] and [15]. The singularity in Lemma 3.7 is not rational, but in this case, the proof of Lemma 3.7 word-for-word works for the family case except that $x_{4} \cdot f\left(x_{0}, x_{1}, x_{2}, x_{3}\right)-x_{0}^{5}=0$ (resp. $\left.\mathbb{C}^{4}\right)$ must be replaced by $F\left(x_{0}, \cdots, x_{4}, t\right)=0\left(\right.$ resp. $\left.\mathbb{C}^{4} \times \Delta\right)$. Therefore, we get a smooth variety $\widetilde{\Gamma}$ by blowing up twice along the 2-dimensional singularity of $\operatorname{Sing}(\Gamma)$ and once for the remaining 1-dimensional singularity of $\operatorname{Sing}(\Gamma)$. Denote by $E$ the exceptional divisor of the last step. We claim that the map $\tilde{\pi}: \widetilde{\Gamma} \rightarrow \Delta$ is a smooth proper submersion. In fact, let $v$ be a vector field of $\Delta$ and let $\tilde{v}$ be a lifting in $\Gamma(\widetilde{\Gamma}, T \widetilde{\Gamma})$ such that $\tilde{\pi}_{*}(\tilde{v})=v$. Denote by $\varphi_{t}\left(\right.$ resp. $\left.\tilde{\varphi}_{t}\right)$ the flow generated by $v($ resp. $\tilde{v})$. Then $\tilde{\varphi}_{t}: \widetilde{\Gamma}^{0} \rightarrow \widetilde{\Gamma}^{t}$ gives the homeomorphism between two fibers of $\tilde{\pi}$ from Ehresmann's Theorem (cf. [16]). This implies the result of the proposition.

From this proposition, we have the following:

Corollary 3.9. For all smooth surfaces $X \subset \mathbb{P}^{3}$ of fixed degree $d=4$, the $\widetilde{\widetilde{V_{3}}}$ thus constructed has a fixed homeomorphism type.

Remark 3.10. A similar statement in Corollary 3.9 holds for arbitrary fixed degree $d$. However, in the general case, the 2 -dimensional singularity $\overline{V_{3}}$ can be resolved by $\left[\frac{d+1}{2}\right]$ blowups (cf. Appendix and [4] for more details). The last proper transform of these blowups has a fixed homeomorphism type.

The proof of Corollary 3.9, For a smooth projective surface $X \subset \mathbb{P}^{3}$, we can find suitable coordinates such that $\left(x_{0}=0\right) \cap X$ is a smooth irreducible curve. In the proof of the proposition, we actually can choose $\tilde{v}$ such that 1) $\tilde{v}$ is tangent to $\Gamma ; 2) \tilde{v}$ is tangent to the exceptional divisor $E$ defined in Proposition 3.8. So the exceptional divisor $E^{t}$ of each fiber at $t \in \Delta$ is the intersection of the fiber $\tilde{\pi}^{-1}(t)$ and the exceptional divisor $E$, i.e., $\tilde{\pi}^{-1}(t) \cap E=E^{t}$. Then the flow of $\tilde{v}$ gives the homeomorphism of any two fibers of $\pi$ since $E$ is smooth.

To see this, let $X, X^{\prime} \subset \mathbb{P}^{3}$ be two smooth hypersurfaces of degree $d$ and let $\underset{\widetilde{V_{3}}}{\approx}\left(\right.$ resp. $\stackrel{\widetilde{V_{3}^{\prime}}}{\approx}$ be the corresponding varieties constructed in Remark 3.6. We also 
denote $W_{3}$ (resp. $\left.W_{3}^{\prime}\right)$ to be the proper transform of $\underset{\overline{V_{3}}}{\approx}\left(\right.$ resp. $\frac{\approx}{\widetilde{V_{3}^{\prime}}}$ ) by resolving the corresponding isolated singular point. (If we use the notation as in the proof of Proposition 3.8 then $W_{3}=\widetilde{\Gamma^{t_{0}}}$ (resp. $W_{3}^{\prime}=\widetilde{\Gamma^{t_{0}^{\prime}}}$ for some $t_{0}, t_{0}^{\prime} \in \Delta$ ).) Then $E^{t_{0}}=E \cap \widetilde{\Gamma^{t_{0}}}=E \cap W_{3}$ (resp. $\left.E^{t_{0}^{\prime}}=E \cap \widetilde{\Gamma^{t_{0}^{\prime}}}=E \cap W_{3}^{\prime}\right)$.

Since the flow $\tilde{\varphi}_{t}$ preserves $E$, the horizonal map $\tilde{\varphi}$ induced by the flow $\tilde{\varphi}_{t}$ in the following diagram

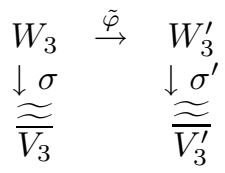

maps $E^{t_{0}}$ onto $E^{t_{0}^{\prime}}$. Hence we have a homeomorphism $g: \underset{\widetilde{V_{3}}}{\approx}\{p\} \cong \underset{\cong}{\widetilde{V_{3}^{\prime}}}-\left\{p^{\prime}\right\}$, where $p\left(\operatorname{resp} . p^{\prime}\right)$ is the corresponding singular point of $\underset{\overline{V_{3}}}{\approx}\left(\operatorname{resp} . \stackrel{\underset{V_{3}^{\prime}}{\approx}}{\approx}\right.$.

Then the homeomorphism $G: \underset{\bar{V}_{3}}{\approx} \underset{\overline{V_{3}^{\prime}}}{\approx}$ is defined by

$$
G(x)= \begin{cases}g(x), & \text { if } x \neq p, \\ p^{\prime}, & \text { if } x=p .\end{cases}
$$

Note that the continuity of $G$ thus defined can be verified by the definition. Indeed, the inverse image of any open set $U^{\prime} \ni p^{\prime}$ under $G$ is $\sigma \circ \varphi^{-1} \circ \sigma^{\prime-1}\left(U^{\prime}\right)$, which is open since $\sigma$ is the quotient map and $E \subset \varphi^{-1} \circ \sigma^{\prime-1}\left(U^{\prime}\right)$.

Therefore, $G$ is a continuous bijective map between compact complex algebraic varieties and so $G$ is a homeomorphism.

We want to show that some Lawson homology group of $\underset{\overline{V_{3}}}{\approx}$ may vary when the smooth $X$ varies in $\mathbb{P}^{3}$.

Theorem 3.11. There exist two rational 3-dimensional projective varieties $Y, Y^{\prime}$ such that $Y$ is homeomorphic to $Y^{\prime}$ but the Lawson homology group $L_{1} H_{3}(Y)$ is not isomorphic to $L_{1} H_{3}\left(Y^{\prime}\right)$ even up to torsion.

Proof. If $X \subset \mathbb{P}^{3}$ is a general smooth quartic surface, then the Picard group $\operatorname{Pic}(X) \cong \mathbb{Z}$ by the Noether-Lefschetz Theorem. For details, see e.g. Voisin [16]. But it is well known that there are still many special smooth quartic surfaces $X^{\prime}$ in $\mathbb{P}^{3}$ such that $X^{\prime} \cap\left(x_{0}=0\right)$ is smooth and $\operatorname{rk}\left(\operatorname{Pic}\left(X^{\prime}\right)\right)$ is as large as 20 . Note that by Theorem 3.1 and the Weak Lefschetz Theorem, $L_{1} H_{2}(X) \cong \operatorname{Pic}(X)$ for any smooth surface $X$ in $\mathbb{P}^{3}$.

Now we choose smooth $X$ with $L_{1} H_{2}(X) \cong \mathbb{Z}$ and $X^{\prime}$ with $L_{1} H_{2}\left(X^{\prime}\right) \cong \mathbb{Z}^{20}$.

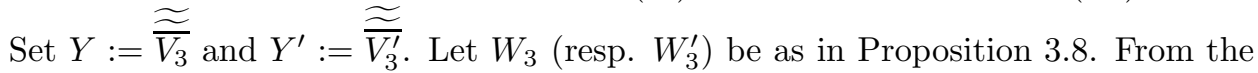
proof of Lemma 2.1 in [8], we have the commutative diagram

$$
\begin{aligned}
& \cdots \rightarrow L_{1} H_{3}(E) \quad \rightarrow \quad L_{1} H_{3}\left(W_{3}\right) \quad \rightarrow \quad L_{1} H_{3}\left(W_{3}-E\right) \quad \rightarrow \quad L_{1} H_{2}(E) \quad \rightarrow \quad \cdots
\end{aligned}
$$



By Lemma 3.7. we know that $E \cong X$. By Theorem 3.1, we have $L_{1} H_{3}(X) \cong$ $H_{3}(X)$. By the Lefschetz Hyperplane Theorem, we know that $X$ is simply connected. Since $q$ is a point, we have $L_{1} H_{3}(Y) \cong L_{1} H_{3}(Y-q) \cong L_{1} H_{3}\left(W_{3}-E\right)$ and $L_{1} H_{2}(Y) \cong L_{1} H_{2}(Y-q) \cong L_{1} H_{2}\left(W_{3}-E\right)$. 
The top row of the above commutative diagram turns into the long exact sequence

$$
0 \rightarrow L_{1} H_{3}\left(W_{3}\right) \rightarrow L_{1} H_{3}(Y) \rightarrow L_{1} H_{2}(X) \rightarrow L_{1} H_{2}\left(W_{3}\right) \rightarrow L_{1} H_{2}(Y) \rightarrow 0 .
$$

Therefore, we have

Since $W_{3}$ is a smooth rational threefold, we have $L_{1} H_{3}\left(W_{3}\right) \cong H_{3}\left(W_{3}\right)$ and $L_{1} H_{2}\left(W_{3}\right) \cong H_{2}\left(W_{3}\right)$ (cf. Prop. 6.16 in [7]). By Proposition 3.8 we have $H_{i}\left(W_{3}\right) \cong$ $H_{i}\left(W_{3}^{\prime}\right)$ for all $i$. Hence from Equation (3.3), we get the following formula for $\operatorname{rk} L_{1} H_{3}(Y)$ :

$$
\operatorname{rk} L_{1} H_{3}(Y)=\operatorname{rk} H_{3}\left(W_{3}\right)+\operatorname{rk} L_{1} H_{2}(X)-\operatorname{rk} H_{2}\left(W_{3}\right)+\operatorname{rk} L_{1} H_{2}(Y) .
$$

By applying Theorem 2.5 to $\left(\widetilde{V_{3}}, D_{1}\right)$, we get

$$
\cdots \rightarrow L_{1} H_{2}\left(D_{1}\right) \rightarrow L_{1} H_{2}\left(\widetilde{\overline{V_{3}}}\right) \rightarrow L_{1} H_{2}\left(\widetilde{\overline{V_{3}}}-D_{1}\right) \rightarrow 0 .
$$

Hence

$$
\begin{aligned}
\operatorname{rk} L_{1} H_{2}\left(\widetilde{\overline{V_{3}}}\right) & \leq \operatorname{rk} L_{1} H_{2}\left(D_{1}\right)+\operatorname{rk} L_{1} H_{2}\left(\widetilde{\overline{V_{3}}}-D_{1}\right) \\
& =\operatorname{rk} L_{1} H_{2}\left(D_{1}\right)+\operatorname{rk} L_{1} H_{2}\left(\overline{V_{3}}-q\right) \\
& =\operatorname{rk} L_{1} H_{2}\left(D_{1}\right)+\operatorname{rk} L_{1} H_{2}\left(\overline{V_{3}}\right) \\
& \left.\leq \operatorname{rk} L_{1} H_{2}\left(D_{1}\right)+1 \quad \text { (Lemma } 3.4\right) .
\end{aligned}
$$

Similarly,

$$
\operatorname{rk} L_{1} H_{2}\left(\widetilde{\widetilde{V_{3}}}\right) \leq \operatorname{rk} L_{1} H_{2}\left(D_{2}\right)+\operatorname{rk} L_{1} H_{2}\left(\widetilde{\overline{V_{3}}}\right) .
$$

Therefore,

$$
\operatorname{rk} L_{1} H_{2}\left(\underset{\overline{V_{3}}}{\approx} \leq \operatorname{rk} L_{1} H_{2}\left(D_{2}\right)+\mathrm{rk} L_{1} H_{2}\left(D_{1}\right)+1\right.
$$

Since $D_{1}$ (also $D_{2}$ ) is isomorphic to a $\mathbb{P}^{1} \cup \mathbb{P}^{1}$-bundle over a smooth curve $C$, it is easy to compute, by using Theorem [2.5 and the Projective Bundle Theorem [6], that

$$
\operatorname{rk} L_{1} H_{2}\left(D_{1}\right) \leq \operatorname{rk} L_{1} H_{2}(C)+2 \cdot \operatorname{rk} L_{0} H_{0}(C)=1+2 \times 1=3 .
$$

Therefore

$$
\operatorname{rk} L_{1} H_{2}\left(\underset{\overline{V_{3}}}{\widetilde{\widetilde{C}}}\right) \leq 3+3+1=7 .
$$

The same computation applies to $\underset{V_{3}^{\prime}}{\widetilde{C}}$, and we get

$$
\operatorname{rk} L_{1} H_{2}\left(\underset{\overline{V_{3}^{\prime}}}{\approx} \leq 3+3+1=7 .\right.
$$

From this together with Equation (3.4), we have

$$
\begin{array}{rlc}
\operatorname{rk} L_{1} H_{3}\left(\widetilde{\overline{V_{3}}}\right) & \leq \quad \operatorname{rk} H_{3}\left(W_{3}\right)+\operatorname{rk} L_{1} H_{2}(X)-\operatorname{rk} H_{2}\left(W_{3}\right)+7 \\
& =\operatorname{rk} H_{3}\left(W_{3}\right)-\operatorname{rk} H_{2}\left(W_{3}\right)+8 \quad\left(\text { since } L_{1} H_{2}(X) \cong \mathbb{Z}\right) .
\end{array}
$$

On the other hand, we have

$$
\begin{array}{rlc}
\operatorname{rk} L_{1} H_{3}\left(\widetilde{\widetilde{V_{3}^{\prime}}}\right) & & \operatorname{rk} H_{3}\left(W_{3}^{\prime}\right)+\operatorname{rk} L_{1} H_{2}\left(X^{\prime}\right)-\operatorname{rk} H_{2}\left(W_{3}^{\prime}\right) \\
& = & \operatorname{rk} H_{3}\left(W_{3}\right)+\operatorname{rk} L_{1} H_{2}\left(X^{\prime}\right)-\operatorname{rk} H_{2}\left(W_{3}\right) \\
& = & \operatorname{rk} H_{3}\left(W_{3}\right)-\operatorname{rk} H_{2}\left(W_{3}\right)+20 \quad\left(\text { since } L_{1} H_{2}\left(X^{\prime}\right) \cong \mathbb{Z}^{20}\right) .
\end{array}
$$

This shows that $L_{1} H_{3}\left(\underset{\overline{V_{3}}}{\approx}\right)$ is not isomorphic to $L_{1} H_{3}\left(\underset{\overline{V_{3}^{\prime}}}{\approx}\right.$. 
3.2. Application to the case $n=3$. With this construction, if we choose $n=3$ and $X \subset \mathbb{P}^{4}$ to be a general hypersurface of degree $d=5$, then $V_{3}=X-X \cap \mathbb{P}^{3}$ and $S:=X \cap \mathbb{P}^{3}$ is a smooth surface in $\mathbb{P}^{3}$.

The proof of Theorem 1.1: By applying Theorem 2.5 to the pair $(X, S)$, we get

$$
\cdots \rightarrow L_{1} H_{3}\left(V_{3}\right) \rightarrow L_{1} H_{2}(S) \rightarrow L_{1} H_{2}(X) \rightarrow L_{1} H_{2}\left(V_{3}\right) \rightarrow 0
$$

The above long exact sequence (3.5) remains exact after being tensored with $\mathbb{Q}$. Note that $L_{1} H_{2}(X) \otimes \mathbb{Q} \supset \operatorname{Griff}_{1}(X) \otimes \mathbb{Q}$ is an infinite dimensional $\mathbb{Q}$-vector space by [2]. Recall that $L_{1} H_{2}(S)$ is finitely generated since $\operatorname{dim} S=2$ by Theorem 2.5 . Hence $L_{1} H_{2}\left(V_{3}\right) \otimes \mathbb{Q}$ is an infinite dimensional $\mathbb{Q}$-vector space. By Proposition 2.7. we have $L_{1} H_{3}\left(V_{4}\right) \otimes \mathbb{Q} \cong L_{1} H_{2}\left(V_{3}\right) \otimes \mathbb{Q}$ is an infinite dimensional $\mathbb{Q}$-vector space.

Note that $Z_{3}=\overline{V_{4}}-V_{4}$ is defined by $\left(x_{5} \cdot f\left(0, x_{1}, \ldots, x_{4}\right)=0, x_{0}=0\right)$ in $\mathbb{P}^{5}$. Let $S^{\prime}=\left(x_{5}=0\right) \cap\left(f\left(0, x_{1}, \ldots, x_{4}\right)=0\right)$ in the hyperplane $\left(x_{0}=0\right) \subset \mathbb{P}^{5}$. It is easy to see that $S^{\prime} \cong S$. Then $Z_{3}=\mathbb{P}^{3} \cup \Sigma_{p}(S)$, where $\Sigma_{p}(S)$ means the join of $S$ and the point $p=[0: \cdots: 0: 1]$. By applying Theorem 2.5 to the pair $\left(Z_{3}, \Sigma S\right)$, we get

Note that $Z_{3}-\Sigma S \cong \mathbb{P}^{3}-S$. Therefore $L_{1} H_{2}\left(Z_{3}\right) \otimes \mathbb{Q}$ is finite dimensional since both $L_{1} H_{2}(\Sigma S) \otimes \mathbb{Q} \cong L_{0} H_{0}(S, \mathbb{Q}) \cong \mathbb{Q}([1])$ and $L_{1} H_{2}\left(\mathbb{P}^{3}-S\right) \otimes \mathbb{Q}$ are. By the same type of argument, we have $L_{1} H_{3}\left(Z_{3}\right) \otimes \mathbb{Q}$ is finite dimensional since both $L_{1} H_{3}(\Sigma S) \otimes \mathbb{Q} \cong L_{0} H_{1}(S, \mathbb{Q})=0$ (note that $S$ is simply connected) and $L_{1} H_{3}\left(\mathbb{P}^{3}-S\right) \otimes \mathbb{Q}$ are.

By applying Theorem 2.5 to the pair $\left(\overline{V_{4}}, Z_{3}\right)$, we have the following long exact sequence:

$$
\cdots \rightarrow L_{1} H_{3}\left(Z_{3}\right) \rightarrow L_{1} H_{3}\left(\overline{V_{4}}\right) \rightarrow L_{1} H_{3}\left(V_{4}\right) \rightarrow L_{1} H_{2}\left(Z_{3}\right) \rightarrow \cdots .
$$

From (3.7), the infinite dimensionality of $L_{1} H_{3}\left(V_{3}\right) \otimes \mathbb{Q}$, and the finite dimensionality of $L_{1} H_{2}\left(Z_{3}\right) \otimes \mathbb{Q}$ and $L_{1} H_{3}\left(Z_{3}\right) \otimes \mathbb{Q}$, we obtain that $L_{1} H_{3}\left(\overline{V_{4}}\right) \otimes \mathbb{Q}$ is an infinitely dimensional $\mathbb{Q}$-vector space. This completes the proof of Theorem 1.1 .

We can continue the procedure. Set $V_{5}:=\mathbb{C}^{5}-V_{4}$. Then $V_{5}$ can be viewed as an affine variety in $\mathbb{C}^{6}$ defined by $x_{6} \cdot\left(x_{5} \cdot f\left(1, x_{1}, \cdots, x_{4}\right)-1\right)-1=0$. Set $Z_{4}=\overline{V_{5}}-V_{5}$, and so on. It can be shown in the same way that $L_{1} H_{3}\left(Z_{4}\right)$ is finitely generated by using Theorem 2.5 and Lawson's Complex Suspension Theorem. Note that $L_{1} H_{4}\left(V_{5}\right) \cong L_{1} H_{3}\left(V_{4}\right)$ by Proposition 2.7 and so $L_{1} H_{4}\left(V_{5}\right)$ is infinitely generated since $L_{1} H_{3}\left(V_{4}\right)$ is.

By applying Theorem 2.5 to the pair $\left(\overline{V_{5}}, Z_{4}\right)$, we get the long exact sequence

$$
\cdots \rightarrow L_{1} H_{4}\left(Z_{4}\right) \rightarrow L_{1} H_{4}\left(\overline{V_{5}}\right) \rightarrow L_{1} H_{4}\left(V_{5}\right) \rightarrow L_{1} H_{3}\left(Z_{4}\right) \rightarrow \cdots .
$$

Since $L_{1} H_{3}\left(Z_{4}\right)$ is finitely generated, we obtain that $L_{1} H_{4}\left(\overline{V_{5}}\right)$ is infinitely generated by Equation (3.8).

Proposition 3.12. In this construction, $L_{1} H_{k}\left(\overline{V_{k+1}}\right)$ is not finitely generated for $k \geq 3$.

By the Complex Suspension Theorem [11], $L_{p+1} H_{2 p+k}\left(\Sigma^{p} \overline{V_{k+1}}\right) \cong L_{1} H_{k}\left(\overline{V_{k+1}}\right)$. Therefore we get:

Theorem 3.13. For integers $p$ and $k$, with $k>0, p>0$, we can find $a$ rational projective variety $Y$ such that $L_{p} H_{2 p+k}(Y)$ is infinitely generated. 
Remark 3.14. If $k=0$ and $p>0$, there also exist projective varieties $Y$ such that $L_{p} H_{2 p}(Y)$ is infinitely generated. This follows from the Projective Bundle Theorem [6] and a result of Clemens [2].

Remark 3.15. All the $Y$ thus constructed above are singular projective varieties. Can one find some smooth projective variety such that the answer to the question (Q) is positive? Yes, we can. By using a completely different method, the author has constructed examples of smooth projective varieties such that $(\mathbf{Q})$ is true (cf. [9]).

Remark 3.16. Note that all $\overline{V_{k+1}}$ are singular rational projective varieties. For smooth rational projective varieties $Y, L_{1} H_{*}(Y) \otimes \mathbb{Q}$ are finite dimensional $\mathbb{Q}$ vector spaces (14]). The author showed that $L_{1} H_{*}(Y)$ are finitely generated abelian groups [8].

\section{ApPEndix}

Let $f\left(x_{0}, \cdots, x_{4}\right)$ be a general homogeneous polynomial of degree 5 , and let $X$ be a hypersurface of degree 6 in $\mathbb{P}^{5}$ given by $F\left(x_{0}, \cdots, x_{5}\right):=x_{5} f\left(x_{0}, \cdots, x_{4}\right)-x_{0}^{6}=0$. It is easy to see from the proof of Lemma 3.5 that the singular point set of $X$ is the union of a smooth 2-dimensional variety $Y$ given by $x_{0}=x_{5}=f\left(x_{0}, x_{1}, \cdots, x_{4}\right)=0$ and an isolated point $q$ defined by $\left\{x_{0}=f=\frac{\partial f}{\partial x_{0}}=\frac{\partial f}{\partial x_{1}}=\frac{\partial f}{\partial x_{2}}=\cdots=\frac{\partial f}{\partial x_{5}}=0\right\}$. It can be calculated that $q=[0: \cdots 0: 1]$. The two dimensional singularity $Y$ of $X$ is a singularity of $A_{5}$-type. Recall that a point $O \in V \subset \mathbb{C}^{3}$ is a singularity of $A_{n}$-type on $V$ if locally $V$ is defined by $x y-z^{n+1}=0$ and $O=(0,0,0) \in \mathbb{C}^{3}$. The reader is referred to [4] for the resolution of singularities of $A_{n}$-type.

Let $\sigma: \widetilde{\mathbb{P}_{Y}^{5}} \rightarrow \mathbb{P}^{5}$ be the blowup of $\mathbb{P}^{5}$ along the surface $Y$ and $\tilde{X}_{Y}$ be the proper transform in the blowup $\widetilde{\mathbb{P}_{Y}^{5}}$. Denote by $E=\mathbb{P}\left(N_{Y / \mathbb{P}^{5}}\right)$ the exceptional divisor of the blowup. Then $D=E \cap \tilde{X}_{Y} \subset \mathbb{P}\left(N_{Y / \mathbb{P}^{5}}\right)$ corresponds to the image of the tangent cones $T_{p} X \subset T_{p}\left(\mathbb{P}^{5}\right)$ in $\mathbb{P}\left(N_{Y / \mathbb{P}^{5}}\right)$ at points $p \in Y$.

Now

$$
T_{p} X=\left\{\sum_{i_{0}+\cdots i_{5}=2} \frac{\partial^{2} F}{\partial x_{0}^{i_{0}} \cdots \partial x_{5}^{i_{5}}} x_{0}^{i_{0}} \cdots x_{5}^{i_{5}}=0\right\}
$$

a degree 2 polynomial in $\mathbb{P}^{5}$. Direct computation shows that

$$
\begin{aligned}
& \left.T_{p} X=\left\{\frac{\partial f}{\partial x_{0}}(p) x_{0} x_{5}+\cdots+\frac{\partial f}{\partial x_{4}}(p) x_{4} x_{5}\right)=0\right\} \\
& \left.=\left(x_{5}=0\right) \cup\left\{\frac{\partial f}{\partial x_{0}}(p) x_{0}+\cdots+\frac{\partial f}{\partial x_{4}}(p) x_{4}\right)=0\right\} .
\end{aligned}
$$

Hence $D=\tilde{X}_{Y} \cap E$ is a fiber bundle over $Y$ with singular conics as fibers. Clearly, $\tilde{X}_{Y}$ is smooth away from $D$ and $\tilde{q}$, where $\tilde{q}=\sigma^{-1}(q)$. Since $D \subset E$ is a 3 -dimensional variety with singular point set $\operatorname{Sing}(D) \cong Y$, we can show that the singularity of $\tilde{X}_{Y}$ is $Y \cup\{\tilde{q}\}$ :

Proposition 4.1. The proper transform $\tilde{X}_{Y}$ is a 4-dimensional variety in $\mathbb{P}\left(N_{Y / \mathbb{P}^{5}}\right)$ with singularity $S \cong Y \cup\{\tilde{q}\}$, where $\tilde{q}$ is an isolated singular point and $Y$ is the singularity of $A_{3}$-type. 
Proof. From the proof of Lemma 3.5, we see that the singular points $S$ of $X$ consist of two components. One is a smooth surface and the other is an isolated point $q$.

Since $f$ is nonsingular on $Y=\left\{x_{0}=f\left(0, x_{1}, \cdots, x_{4}\right)=0\right\}$, we have $d f \neq 0$ on $Y$. Let us restrict ourselves to a neighborhood of a point $p$ in $Y$. There, we can take the neighborhood of $p$ as the affine space $\mathbb{C}^{5}$ with $p$ the origin. Hence we can choose $y=f$ as a coordinate in the neighborhood of each point on $Y$ since it is smooth. Locally, $Y$ is defined by $x_{0}=x_{5}=y=0$ in $\mathbb{C}^{5}$. We denote it by $Y_{0}$. For convenience, we denote by $x=x_{0}$ and $z=x_{5}$ the other two normal coordinates to



$$
\left\{\begin{array}{l}
x v=u y, \\
x w=u z, \\
y w=z v
\end{array}\right.
$$

in $\mathbb{C}^{5} \times \mathbb{P}^{2}$, where $[u: v: w]$ is the homogeneous coordinate on $\mathbb{P}^{2}$. Let $\left.\sigma: \widetilde{\left(\mathbb{C}^{5}\right.}\right)_{Y_{0}} \rightarrow$ $\mathbb{C}^{5}$ be the map of this blowup. Then the inverse image of $X$ is given by the following equations:

$$
\left\{\begin{array}{l}
x^{6}-y z=0, \\
x v=u y \\
x w=u z \\
y w=z v .
\end{array}\right.
$$

The above equations define two divisors on $\widetilde{\left(\mathbb{C}^{5}\right)_{Y_{0}}}$. One of them is the exceptional divisor $E_{0}$, the intersection of $E$ with $\left(\mathbb{C}^{5}\right)_{Y_{0}}$ and the other is exactly the proper transform $\widetilde{X}_{Y_{0}}$ of $X_{0}$ in $\left.\widetilde{\mathbb{C}^{5}}\right)_{Y_{0}}$, where $X_{0}$ is the part of $X$ in $\mathbb{C}^{5}$.

We want to show that $\widetilde{X}_{Y_{0}}$ is smooth away from $Y_{0}$. Now it is clear. The blowup $\left.\widetilde{\left(\mathbb{C}^{5}\right.}\right)_{Y_{0}}$ is covered by 3 open charts: $(u \neq 0),(v \neq 0)$ and $(w \neq 0)$.

On the chart $(u \neq 0)$, we can set $u=1$. The equations for the inverse image of $X_{0}$ under $\sigma$ are given by

$$
\left\{\begin{array}{l}
x^{6}-y z=0 \\
x v=y \\
x w=z \\
y w=z v
\end{array}\right.
$$

The equations $x v=y$ and $x w=z$ imply $y w=z v$. Replacing $y$ and $z$ by $x v$ and $x w$, respectively, we can factor $x^{2}$ in the first equation $x^{6}-(x v)(x w)=0$. Hence the proper transform $\widetilde{X}_{Y_{0}}$ is given by

$$
\left\{\begin{array}{l}
x^{4}-v w=0, \\
x v=y, \\
x w=z,
\end{array}\right.
$$

and the exceptional divisor for $\widetilde{X}_{Y_{0}} \rightarrow X_{0}$ is given by

$$
\left\{\begin{array}{l}
x^{2}=0, \\
x^{4}-v w=0, \\
x v=y, \\
x w=z ;
\end{array}\right.
$$

i.e., $x=y=z=v=0$ or $x=y=z=w=0$. Each component is a holomorphic line over $(x=y=z=v=w=0) \cong Y_{0}$. 
From the definition, the singularity of $\widetilde{X}_{Y_{0}}$ is given by equations $x^{4}-v w=$ $0, y-x v=0, z-x w=0$ and

$$
\operatorname{rank}\left(\frac{\partial\left(x^{4}-v w, y-x v, z-x w\right)}{\partial(x, y, z, v, w)}\right) \leq 2,
$$

which is $x=y=z=v=w=0$; i.e., it is isomorphic to $Y_{0}$.

A similar calculation works for the charts $(v \neq 0)$ and $(w \neq 0)$, and the proper transform $\widetilde{X}_{Y_{0}}$ is smooth everywhere. This completes the proof of the proposition.

Denote by $\tilde{Y}$ the 2-dimensional singularity of $\widetilde{X}_{Y}$. Let $\tilde{\sigma}: \widetilde{\widetilde{\mathbb{P}_{Y}^{5}}} \rightarrow \widetilde{\mathbb{P}_{Y}^{5}}$ be the blowup of $\widetilde{\mathbb{P}_{Y}^{5}}$ along $\tilde{Y}$. Let $\widetilde{\widetilde{X}}_{Y}$ be the proper transformation of $\widetilde{X}_{Y}$ under $\tilde{\sigma}$. Set $\tilde{\tilde{q}}=\tilde{\sigma}^{-1}(\tilde{q})$.

Proposition 4.2. The singularity of the proper transform $\widetilde{\widetilde{X}}_{Y}$ is isomorphic to $Y \cup\{\tilde{\tilde{q}}\}$, where $\tilde{\tilde{q}}$ is an isolated singular point and the 2-dimensional singularity is of $A_{1}$-type.

Proof. One blowup at the singularity reduces the singularity of $A_{n}$-type to $A_{n-2^{-}}$ type (cf. [4]). A detailed computation from $A_{5}$-type to $A_{3}$-type has been given in Proposition 4.1 .

Remark 4.3. The 2-dimensional singularity of $X$ can be resolved by blowing up one more time. In general, an $A_{n}$-type singularity can be resolved by blowing up $\left[\frac{n+1}{2}\right]$ times (cf. 4]). The 1-dimensional singularity in the example we used in subsection 3.1 is of $A_{4}$-type, as stated in Remark 3.6. so it can be resolved by two blowups. The isolated singularity $q$ can be resolved by one blowup, as we have done in Lemma 3.7

\section{ACKNOWLEDGEMENTS}

I would like to express my gratitude to my former advisor, Blaine Lawson, for proposing these problems and for all his help. I also would like to thank the referee for correcting some mathematically imprecise terms as well as writing and typos in the original version.

\section{REFERENCES}

1. E. Brieskorn, Singular elements of semi-simple algebraic groups, Actes du Congrès International des Mathématiciens (Nice, 1970), Tome 2, Gauthier-Villars, Paris, 1971, pp. 279-284. MR0437798 (55:10720)

2. Herbert Clemens, Homological equivalence, modulo algebraic equivalence, is not finitely generated, Inst. Hautes Études Sci. Publ. Math. (1983), no. 58, 19-38 (1984). MR720930 (86d:14043)

3. Albrecht Dold and René Thom, Quasifaserungen und unendliche symmetrische Produkte, Ann. of Math. (2) 67 (1958), 239-281. MR0097062 (20:3542)

4. Alan H. Durfee, Fifteen characterizations of rational double points and simple critical points, Enseign. Math. (2) 25 (1979), no. 1-2, 131-163. MR.543555 (80m:14003)

5. Eric M. Friedlander, Algebraic cycles, Chow varieties, and Lawson homology, Compositio Math. 77 (1991), no. 1, 55-93. MR1091892 (92a:14005)

6. Eric M. Friedlander and Ofer Gabber, Cycle spaces and intersection theory, Topological methods in modern mathematics (Stony Brook, NY, 1991), Publish or Perish, Houston, TX, 1993, pp. 325-370. MR 1215970 (94j:14010) 
7. Eric M. Friedlander, Christian Haesemeyer, and Mark E. Walker, Techniques, computations, and conjectures for semi-topological K-theory, Math. Ann. 330 (2004), no. 4, 759-807. MR2102312 (2006c:19004)

8. Wenchuan $\mathrm{Hu}$, Birational invariants defined by Lawson homology, arXiv:math/0511722, to appear in Int. J. of Pure and Appl. Math.

9. —— Generalized Abel-Jacobi map on Lawson homology, arXiv:math/0608294, August 2006.

10. János Kollár, Yoichi Miyaoka, and Shigefumi Mori, Rationally connected varieties, J. Algebraic Geom. 1 (1992), no. 3, 429-448. MR.1158625 (93i:14014)

11. H. Blaine Lawson, Jr., Algebraic cycles and homotopy theory, Ann. of Math. (2) 129 (1989), no. 2, 253-291. MR 986794 (90h:14008)

12. 1993), Int. Press, Cambridge, MA, 1995, pp. 137-213. MR1375256 (97m:14006)

13. Paulo Lima-Filho, Lawson homology for quasiprojective varieties, Compositio Math. 84 (1992), no. 1, 1-23. MR1183559 (93j:14007)

14. C. Peters, Lawson homology for varieties with small Chow groups and the induced filtration on the Griffiths groups, Math. Z. 234 (2000), no. 2, 209-223. MR1765879 (2001f:14042)

15. G. N. Tjurina, Resolution of singularities of flat deformations of double rational points, Funkcional. Anal. i Priložen. 4 (1970), no. 1, 77-83. MR0267129 (42:2031)

16. Claire Voisin, Théorie de Hodge et géométrie algébrique complexe, Cours Spécialisés [Specialized Courses], vol. 10, Société Mathématique de France, Paris, 2002. MR1988456 (2005c:32024a)

Department of Mathematics, Massachusetts Institute of Technology, Building 2, Room 363B, 77 Massachusetts Avenue, Cambridge, Massachusetts 02139-4307

E-mail address: wenchuan@math.mit.edu 